\title{
Design and Control of a High-Efficiency Doubly-Fed Brushless Machine for Wind Power Generator Application
}

\author{
Longya Xu, Bo Guan, Huijuan Liu, Le Gao, and Kaichien Tsai \\ Dept. of Electrical and Computer Engineering \\ The Ohio State University, Columbus, OH 43210 \\ The Ohio State University
}

\begin{abstract}
Principles and latest progress of Doubly Fed Brushless Machine (DFBM) are reviewed with the identified solutions to designing a high efficiency DFBM of $96 \%$. The original design of DFBM rated at $200 \mathrm{kw} / 1200 \mathrm{rpm}$ focuses on the key components of DFBM, including the rotor style and pole number combination, and control algorithms. Both theoretical and experimental results are presented to substantiate the success of the original design solutions.
\end{abstract}

Keywords- doubly fed, brushless, high efficiency, decouple control of active and reactive power, wind power generator

\section{INTRODUCTION}

The basic idea of doubly-fed brushless machine (DFBM) can be traced back nearly 100 years and has experienced three major development stages. For the very early and first stage around 1910-1920s, Hunt and Creedy researched the concept of "self-cascaded induction motor". Stemming from the cascaded induction machine, the "self-cascaded induction motor" essentially is two wound induction motors in a special arrangement. Mechanically the two induction motors share a common shaft and, at the same time, electrically the two sets of rotor windings are cascaded [1,2]. As a result, the overall system was left with two sets of terminals for the two sets of stator windings. Since the two sets of rotor windings are selfcascaded, no brushes and slip rings are needed as shown in Fig. 1.

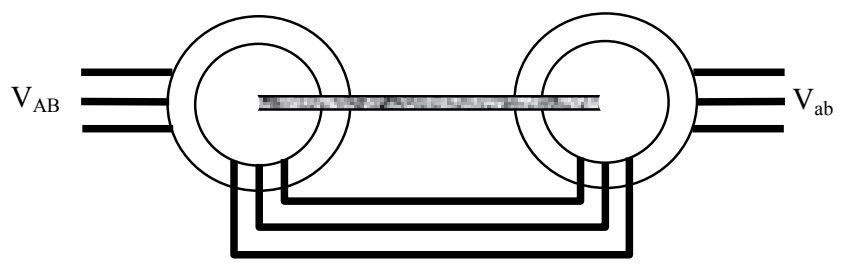

Fig. 1. Two induction machines in cascade connection

High starting torque and speed regulation were obtained with certain success. With the help of power electronics control, this early version of DFBM, with minor variations, still finds application nowadays [3]. While 50 years later coming the second stage around 1970s, Broadway, Thomas, Kusko, Somuah and others conducted further research and published their in-depth understanding in the concept of "selfcascaded induction motor" [4-6]. It was proposed to merge the two sets of stator windings by a dual-tapped stator windings wound into a common stator core. Also, it was proposed to design a special rotor common to the dual-tapped stator windings. In this way, the early version of DFBM was evolved from "self-cascaded induction motor" based on two separated machines to a single electric machine. However, in terms of modeling and analysis of the machine, all researchers during that period still treated the machine as two separate machines but built into a single frame. Along the way, creative ideas sprouted and of particular mentioning was Broadway's rotor in two styles, the nested cage rotor and the salient reluctance rotor that could be equally effective for the single-frame "self-cascaded induction motor" [4]. With complicated derivations, Broadway gave steady-state equations and equivalent circuit for the self-cascaded machine, and named it "Brushless Stator-Controlled SynchronousInduction Machines" [5]. At the beginning of 80 s one attempt was made by Heyne and El-Antably to build the Broadway's version of DFBM [6]. This prototype, perhaps, is the first ever detailed experimental investigation which, to certain degree, has verified the basic principles but did not produce meaningful torque density and energy efficiency for practical applications. In addition, control principles were to be developed and algorithms implemented.

The renewed interests in DFBM in 1990s, regarded as the third stage with much strength, was pushed by the rapid advances in modern power electronics which, supposedly, can take full advantages of possible potentials of the DFBMs in variable speed drives and variable-speed constant-frequency generators. Many papers have been published continuing the discussions in the DFBM principles, operational characteristics, and possible advanced control methods and applications. The most important development in this stage is that researchers have formally established the identity of DFBM as a special machine, instead of treat it as two machines in "self-cascade", that is, a combination of two induction machines built on a common shaft or single stator house [9-12]. Through their research work, it was established that, regardless rotor types, the DFRM terminal characteristics resemble those of a wound rotor induction machine. Detailed field analysis of DFBM further verified that the two sets of stator windings in a DFBM resemble the mutual coupling of the stator and rotor windings in a conventional wound induction machine. However, from the structure robustness point of view, the attractiveness of the DFBM machine is 
evident - the machine can be operated as a conventional doubly fed induction machine but eliminate all the headaches associated with brushes and slip rings, from high costs of building and maintaining the machine to the serious reliability issues. Attracted by the features of brushless and doubly fed operation modes, several prototype DFBMs were built and tested. Unfortunately, the experiment results did not show a great promise with efficiency only about $75 \%$ and inability to reach the designed full power [13-14].

Nevertheless, the research results in the last 20 years did reveal clearly a series of fundamental issues and challenges with respects to the design and control a DFBM. Compared to its counterpart with brushes/slip-rings, the issues of DFBM to the researchers are fundamental and critical, including a) what are the rules for DFBM optimal design to maximize torque and power density, b) what are the suitable control algorithms for a DFBM system, c) how the energy efficiency can be substantially improved, and d) what are the ultimate limits on design and control such a machine. Evidently, these challenges are of practical significance and application demanding. Without innovative breakthroughs to the issues, the dreamed Doubly-Fed Brushless Machine technology would remain as a dream on academic papers.

This paper presents our latest investigation of optimal design and advanced control of DFBM by an original design of $200 \mathrm{kw} / 1200 \mathrm{rpm}$ for high-efficiency and -reliability wind power generator application. The paper starts with a brief review of main configuration and basic principles of DFBM and system. Then, the challenges of design a high-efficiency DFBM and system are highlighted. Following the challenge description are the identified solutions to the optimal design and control algorithms. Using finite element analysis, the paper presents the original design of DFBM and system in a power rating of $200 \mathrm{kw}$ for a speed range of 400-1200rpm. The designed machine is built and tested in laboratory, and the experimental results are presented. It is shown that the newly designed DFBM can achieve an energy-efficiency as high as $96 \%$ over a large operational region. The breakthrough achievements in high efficiency are discussed with remaining challenges described. Future work to improve DFBM machine and system is recommended.

\section{DOUBLY FED BRUSHLESS MACHINE AND SYSTEM}

\section{A. DFBM Structure and System Configuration}

A DFBM variable speed drive (generating) system as shown in Fig. 2 consists of three main components, a DFBM, a back-to-back converter and an associated controller. DFBM is a controlled electric machine, meaning that for its practical applications, it is necessary to mate the DFBM with a bidirectional power flow converter.

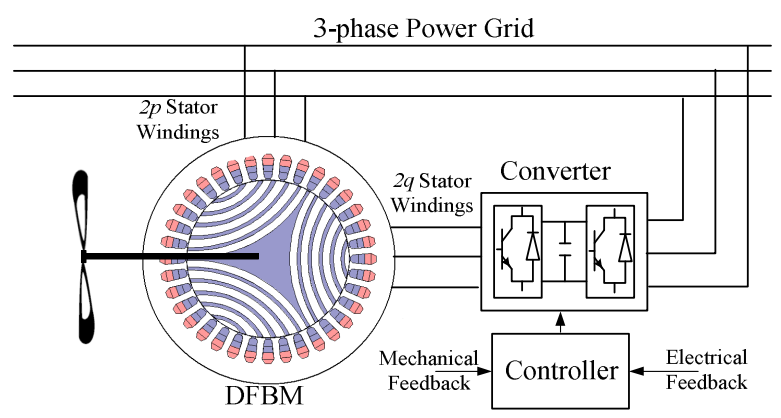

Fig. 2 Schematic of DFBM system

As shown, the stator of a DFBM has two sets of 3-phase sinusoidally distributed windings. One set of windings, the primary, is connected directly to the power grid. The other set, the secondary, is fed with variable voltages at variable frequencies from a converter that also is connected to the power grid. When both sets of stator windings are fed from a set of 3-phase symmetric currents, two rotating MMFs are produced along the DFBM air-gap. Since the two sets of windings differ in pole numbers, one of $2 p$ and another $2 q$, and are excited with currents of different frequency, one of $\omega_{1}$ and another $\omega_{2}$, the two stator rotating MMFs differ from each other. According to electric machine fundamentals, under normal conditions, the two stator MMFs have no useful interaction for electromechanical energy conversion, except for torque pulsations. However, in the structure as described, with the magnetic modulation of the rotor, the two MMFs do have useful interaction for electromechanical energy conversion.

The rotor of the DFBM can be built in one of the two styles: the rotor with nested cages of $p_{\mathrm{r}}$ circuits or rotor with reluctance segments of $p_{\mathrm{r}}$ pieces where

$$
p_{\mathrm{r}}=p+q
$$

Eq. (1) indicates that $p_{\mathrm{r}}$, the number of rotor nested cage circuits or reluctance segments, is constrained by the pole numbers of the two sets of stator windings. Eq. (1) also simply states that among the three numbers $\left(p_{r}, p\right.$ and $\left.q\right)$ we can independently choose two (two degrees of freedom) and the third is determined by the equation. The operation of the DFBM relies on the interaction of the two stator MMFs through modulation action of the rotor. When one set of symmetrical sine-wave currents of frequency $\omega_{1}$ are flowing in the primary windings, three-phase back EMFs will be induced with a frequency of $\omega_{2}$ in the secondary windings and vice versa. The two electrical frequencies, $\omega_{1}$ and $\omega_{2}$, in the primary and secondary are related to the rotor mechanical speed $\omega_{\mathrm{rm}}$ by

$$
\omega_{\mathrm{rm}}=\left(\omega_{1}-\omega_{2}\right) / p_{\mathrm{r}}
$$


Electromechanical energy conversion will take place in DFBM if Eq. (2) is satisfied. Eq. (2) clearly shows that among the three speeds $\left(\omega_{1} \omega_{2}\right.$ and $\left.\omega_{\mathrm{rm}}\right)$, we can independently control two and the third is determined by the equation. In motor operation for speed control, a predetermined rotor speed $\omega_{\mathrm{rm}}$ is achievable if $\omega_{1}$ and $\omega_{2}$ are controlled. As in generator operation, variable-speed constant-frequency generation is achievable by DFBM if $\omega_{1}$ or $\omega_{\mathrm{rm}}$ are predetermined with $\omega_{2}$ controlled conforming to Eq. (2).

Depending on the sequence and value of the controlled frequency $\omega_{2}$, a DFBM can operate in different modes. In particular, in the doubly-fed mode with $\omega_{2}=0$, the DFBM is operated as a synchronous machine. On the other hand, with $\omega_{2}>0$ (positive sequence) or $\omega_{2}<0$ (negative sequence), a DFBM can operate below or above its synchronous speed. In terms of power flow, regardless at the sub-, super-, or synchronous rotor speed, DFBM always can be operated as a motor or generator. For the above arguments, it is more reasonable just to name this machine "doubly-fed brushless machine" or DFBM than to call it doubly fed brushless "cage induction" or "reluctance" machine, as in the past literature. The machine is not called "cage" or "reluctance" machine because it is so much different from conventional cage induction and synchronous reluctance machines in the traditional and classical sense of cage or reluctance machine.

\section{B. Torque Density}

In our previous publication, it was made clear by both the linear and non linear models that for a DFBM, when one set of stator windings is excited, back EMFs will be induced in the other set of stator windings of different pole numbers, due to rotation of the rotor [12]. The induced EMFs under the influence of a moving rotor are the keys for DFBM to accomplish electromechanical energy conversion because the induced voltage so-achieved is called "speed voltage". Assuming that a set of currents of the same frequency as that of the "speed voltage" are injected into the second set of stator windings, we see that electromechanical power and electromagnetic torque takes place. The general form of the torque production in DFBM is

$$
\begin{aligned}
T_{e}= & \frac{3 E_{p} I_{p} \cos \phi+3 E_{q} I_{q} \cos \phi}{\omega_{r m}}=\frac{3 \omega_{1} \lambda_{m q} I_{p} \sin \phi+3 \omega_{2} \lambda_{m p} I_{q} \sin \phi}{\omega_{r m}} \\
= & \frac{3}{2}(p+q) \lambda_{m q} I_{p} \sin \phi
\end{aligned}
$$

where the $E_{\mathrm{p}}$ and $E_{\mathrm{q}}$, are the induced back EMFs, the speed voltages associated with the variations of the mutual flux linkages; $I_{\mathrm{p}}$ and $I_{\mathrm{q}}$ the phase currents; $\phi$ the angle between the induced speed voltage and the current; and $\lambda_{\mathrm{mp}}$ and $\lambda_{\mathrm{mq}}$ the mutual flux linkages under the given currents. Note that in the torque derivation, the relation $\lambda_{\mathrm{mp}} / \lambda_{\mathrm{mq}}=I_{\mathrm{p}} / I_{\mathrm{q}}$ is used and the frequency constrain in Eq. (2) observed.

Examining Eq. (3), we can relate the torque production to the DFBM magnetic structure. Note that in the equation, the currents, $I_{\mathrm{p}}$ and $I_{\mathrm{q}}$, and phase angle $\phi$ are externally controlled variables and only the mutual flux linkages, $\lambda_{\mathrm{mp}}$ and $\lambda_{\mathrm{mq}}$ are DFBM parameter (mutual inductance) or its magnetic structure related. According to the electric machine fundamentals, the mutual inductance between two windings in an electric machine is proportional to the winding turn numbers and the magnetic permeance of the magnetic flux paths. If the DFBM stator windings and iron laminations are of a traditional design as those in any traditional machine, we are left with choices only in the DFBM rotor design. It is extremely important to realize that the function of the DFBM rotor is to modulate one MMF (for example 6-pole MMF) so as to create the largest possible flux linkages compatible to another windings of different pole number (for example, 4pole windings). It is clear that the larger the rotor modulation capability, the better the rotor design and the larger mutual inductance between two sets of stator windings.

Our previous investigations on rotor styles indicated that the rotor style has strong effects on the rotor modulation capabilities. For the rotor with nested cage circuit, the modulation effects are medium and rotor currents in the nested cage circuits are necessary and, hence, conduction losses are inevitable. On the other hand, for the current-free reluctance segmental rotor, the modulation capabilities are diverse, depending on the reluctance structures. Three types of current-free reluctance rotors were investigated in detail, including simple salient rotor, axially laminated rotor and magnetically isolated segmental rotors [13]. It is discovered that the simple salient rotor, through robust and low cost, the modulation capability is very poor and has parasitic pole numbers in additional to the intent segmental number $p_{r}$. Therefore, the simple salient pole rotor is of little use in DFBM design. The axially laminated rotor showed very promising magnetic modulation, creating the largest mutual coupling between the two stator windings. One major concern with the axially laminated rotor, however, is its inability to block eddy-current circulation in the planes vertical to the magnetic flux lines that continuously and radically change due to the rotor's magnetic modulation. In addition, the resultant eddy currents can cause serious problems for the DFBM: a) heavy eddy current losses; and b) distorted flux line distribution pushed by the large eddy currents. Based on the above considerations, we identified that innovative approaches are needed to design the rotor that achieves both strong rotor modulation and free of eddy current losses.

In addition to the rotor style selection and laminations design, we also found that the combinations of the DFBM pole number are of great influence to the rotor modulation and, 
thus, to the mutual coupling of the two stator windings. In [14], three cases of pole number combinations are investigated using the same stator frame and lamination structures: a) $p=1$, $q=2$ and $p_{r}=3$; b) $p=1, q=3$ and $p_{r}=4$; and c) $p=2, q=3$ and $p_{r}=5$. Finite element analysis conducted for the conditions of the same copper losses and results indicated that the torque production for $p_{r}=5$ is $40-50 \%$ more than those achieved in the other two cases. The strong impact of the DFBM pole number combination on DEBRM is obvious.

\section{Energy Efficiency}

To maximize energy efficiency of a DFBM is equivalent to minimizing various losses, mainly the iron and copper losses. Considerations of minimizing copper losses immediately exclude the selection of a rotor with nested cage circuits. This is because for the DFBM with the nested cage rotor, the modulation function of the rotor is obtained at the costs of currents in the nested cages, inherently causing significant conduction losses. If the current-free reluctance rotor is chosen, the rotor will be free of copper losses while achieving modulating the two stator MMFs.

While minimizing copper losses favors current-free reluctance rotor, we still face the choices among the various reluctance structures: simple saliency, isolated segments, axially laminated and others. As discussed before, we can easily eliminate the simple salient rotor for its poor modulation capability. If we turn to the axially laminated rotor for greater modulation capability, the eddy current losses become unacceptable. It is logical that we choose a normally laminated rotor in segments over axially laminated rotor if equal or stronger modulation capability is achievable. Detailed design approach of a normally laminated rotor is given in the next section.

\section{DESIGN AND PERFORMANCE PREDICTION}

\section{A. DFBM Sizing}

The sizing of the 200kw/1200rpm DFBM starts with a comparable and conventional doubly fed wound rotor induction machine. Since the DFBM to be designed relies on the rotor modulation to create mutual coupling between the two sets of stator windings of different pole numbers, we have purposely enlarged the frame size by $40 \%$, compared to that of the conventional doubly fed induction machine. By try-anderror, the DFBM machine main dimensions are determined as listed in Table 1, together with the DFBM specifications.

\begin{tabular}{||l|c|l|c||}
\hline \hline Power $(\mathrm{kW})$ & 200 & rated rpm & 1200 \\
\hline $\mathrm{V}_{\text {line }}($ volts, rms $)$ & 690 & rated hz & 50 \\
\hline $\mathrm{I}_{\mathrm{P}}(\operatorname{amps}, \mathrm{rms})$ & 125.5 & $1^{\text {st }}$ winding & $3 \phi, 6$-pole \\
\hline $\mathrm{I}_{\mathrm{S}}($ amps, $\mathrm{rms})$ & 125.5 & $2^{\text {nd }}$ winding & $3 \phi, 4$-pole \\
\hline Power Factor & variable & rotor segts & 5 \\
\hline \multicolumn{3}{|l}{} \\
\hline
\end{tabular}

\begin{tabular}{||l|c|l|c|}
\hline Stator OD $(\mathrm{mm})$ & 740 & Length $(\mathrm{mm})$ & 600 \\
\hline Stator ID $(\mathrm{mm})$ & 501 & stator lamination & DW360 \\
\hline Rotor OD $(\mathrm{mm})$ & 499.4 & rotor lamination & DW360 \\
\hline
\end{tabular}

Table 1 Specifications and main dimensions

As summarized in the table, the DFBM is designed with two sets of stator windings, one for 6-pole and another 4-pole. The synchronous speed of the designed DFBM $600 \mathrm{rpm}$ and, in order to deliver the rated power of $200 \mathrm{kw}$, the DFBM needs to run at a super synchronous speed of 1,200rpm.

\section{B. Segmental Rotor Design}

A single sheet of rotor lamination for one rotor segment is shown in Fig. 3. As indicated, the rotor is designed with five, laminated rotor segments and each segment is formed by a stack of radial laminations with a proper pressure, as shown in the figure. The designed laminations in the radial direction serves two purposes: a) to force the magnetic flux lines travelling along the five paths imposed by the designed magnetic paths; b) to minimize the eddy-current losses by orienting lamination parallel to the magnetic flux lines. The ratio of air space to the width of the magnetic path is carefully kept at 2:3 so that when the full load currents apply, the iron materials do not saturate to guarantee strong modulation capabilities of the rotor.

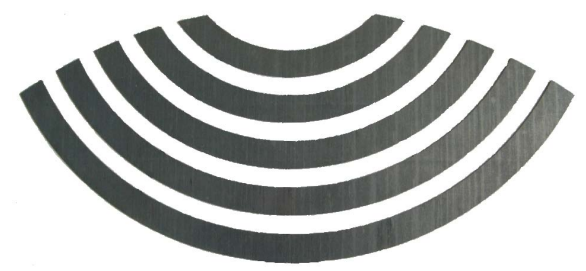

Fig. 3 Lamination for rotor segments

It is noticeable that the rotor designed ensures the entire rotor body nearly non-electrical and non-magnetic conductive except for the magnetic paths designed on purpose. This guarantees that any magnetic and electric short circuits are not allowed so as not to misguide the flux lines and degrade the DFBM power density and energy efficiency. Precautions also have been taken in the rotor design to minimize all possible losses. Additionally, non-electrical and-magnetic conductive epoxy bonding materials are chosen to hold all laminated segments together for rotor mechanical integrity and proper heat rejection.

\section{Performance Prediction}

As the final step in design, we have used finite element analysis to verify three major design objectives: a) mutual coupling and induced back EMFs; b) torque production under various controlled conditions, and c) magnetic field distribution and magnetic loading under no load and loaded conditions. As shown in Fig. 4, the magnetic field of the 
DFBM is not symmetric and computation of the entire magnetic field for the DFBM is needed.

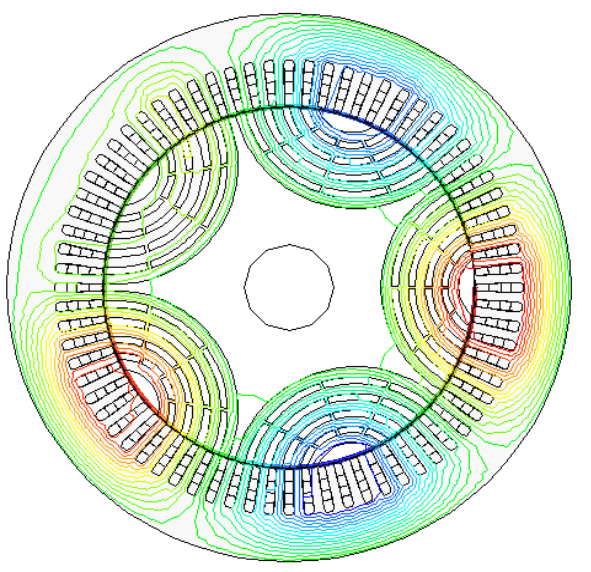

Fig. 4 None-symmetric rotor magnetic structure

For the verification of the first objective, only one of the two sets of stator windings are excited and the induced back EMF voltages are computed for another set stator windings based on the winding flux linkage variations as the rotor positions change. This calculation is repeated at many current levels close to iron core saturation. All calculation results of induced voltage are summarized into the magnetizing curves shown in Fig. 5. The results show that the two sets of stator windings do emulate the functions of the stator and rotor windings respectively in a conventional doubly fed induction machine, that is, when one set of windings is excited, an induced speed voltage occurs in another set of windings. In addition, the relationship among the three frequencies constrained by Eq. 2 is fully verified.

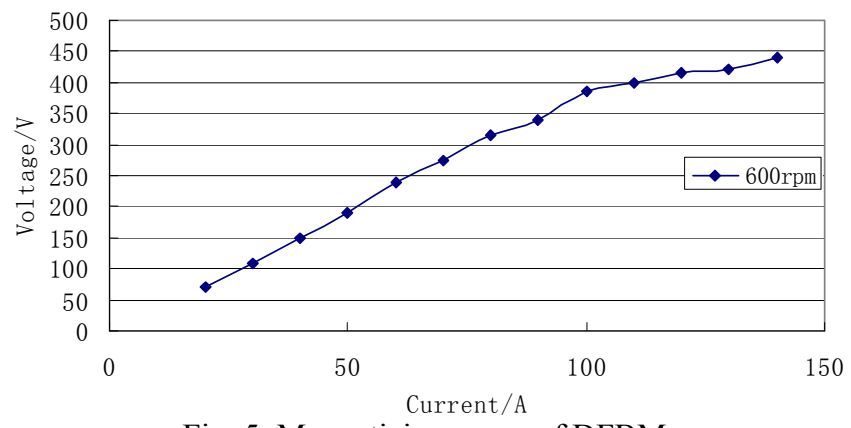

Fig. 5 Magnetizing curve of DFBM

For the verification of the second design objective, finite element analysis is used to calculate torque production. In calculation, both sets of the stator windings are excited with the frequencies conforming to Eq. 2. At the same time the relative phase angles are changed as a parameter of the torque production. The torque computation by finite element analysis is directly based on the magnetic flux density and field intensity on each element. The results are considered accurate because the complicated geometry of the DFBM and nonlinearity of the materials are taken into full account. The torque production of the DFBM as a function of the phase angle between the two sets of stator windings are shown in Fig. 6 for the rated current levels listed in Table 1.

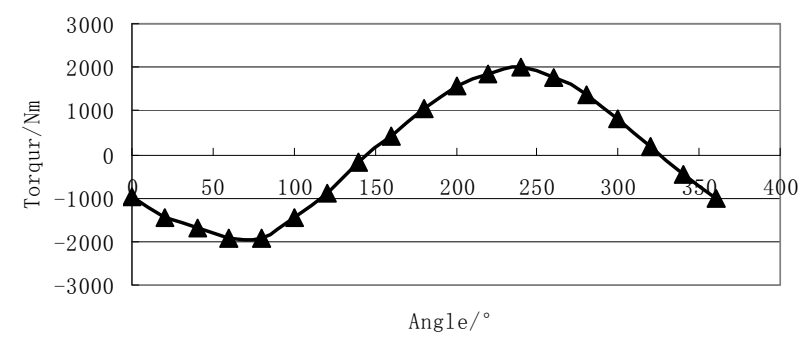

Fig. 6 Torque Capability of DFBM with Rated Currents

Verified by the results are that the rated torque is fully achievable and so is the designed power capability. Another result we can derive at the rated power level is the copper losses for the proportional amount of torque. For the rated torque condition $(1600 \mathrm{Nm})$, the copper losses of both stator windings are about $3 \mathrm{kw}$, less than $2 \%$ of the rated output power.

For the verification of the third design objective, finite element analysis is used to plot the magnetic field distributions for typical no load and loaded conditions. The difficulties in predicting iron core losses are substantial since the magnetic field distributions in space and variations in time of DFBM are not sinusoidal as compared to those in a conventional AC machine. We have investigated many locations across all iron core area to identify magnetic saturation spots and magnetic field variation patterns. The most important observation is the unsymmetrical distribution of air-gap flux density as shown in Fig. 7. In the figure, it displays that the five rotor segments conduct magnetic flux lines in different amount, which forms unsymmetrical magnetic distribution. The unsymmetrical magnetic distribution implies unbalanced pulling force in the various radial directions and non-uniformed torque force distribution along the DFBM air gap. It is suggested by Fig. 7 that unbalance radial forces be investigated by experimental testing to verify theoretical computation.

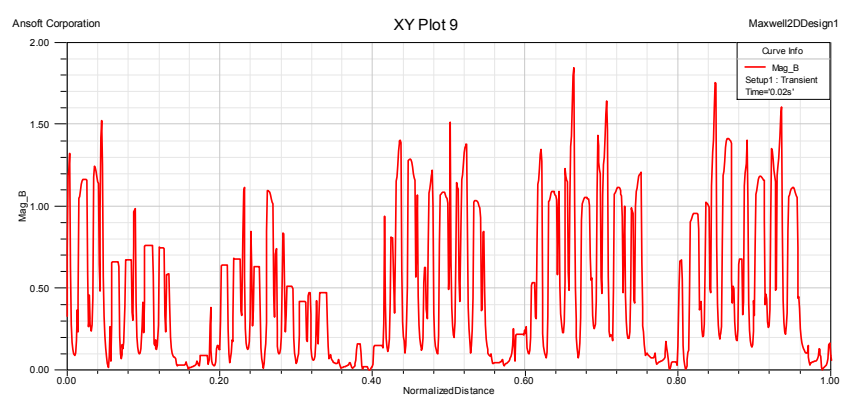

Fig. 7 Magnitude of air-gap flux density distribution 


\section{CONTROL STRATEGY}

The control strategy of the designed DFBM is developed based on the features of a doubly fed system illustrated in Fig. 2. One set of the stator windings is constantly connected to the power grid with a fixed voltage and constant frequency, and the other to a converter with variable voltage and frequency, adapted to the rotor speed. We can improve current utilization of the DFBM by the locking the phase angle of the two sets of stator current to a special angle to realize the so-called vector control principles.

Another less obvious control requirement for DFBM is that the two sets of stator windings are of different pole-pitch and excited with currents of different frequencies, which implies that the same space angle in the DFBM air-gap means different electrical angles to the two sets of stator windings. The equivalence of the angle transformation, however, can be obtained by integrating both sides of Eq. 2, resulting in

$$
\theta_{\mathrm{rm}}=\left(\theta_{1}-\theta_{2}\right) / p_{\mathrm{r}}
$$

Eq. 4 plays the key role to translate the three different phase angles into a uniform scale and then field orientation or vector control is applied to the DFBM. If the in-phase component of the current with respect to the induced speed voltage is controlled, so is the torque production and real power of the DFBM. On the other hand, if the current component orthogonal to the induced speed voltage mutual is controlled, so is the reactive power and, thus, the terminal power factor. It is no surprising that DFBM, like its brush type counterparty, can be conveniently used to achieve decoupled control of active and reactive power, a very useful feature for variable-speed constant-frequency in wind turbine generator application in which the active power often needs to be controlled according to the wind blow speed.

\section{EXPERIMENTAL TESTING RESULTS}

The designed 200kw/1200rpm DFBM was built and tested in our laboratory. The photos of the DFBM stator, rotor and total assembly are shown in Fig. 8. As can be observed, the stator terminals are doubled because of the dual stator windings while the rotor is current-free with no windings, brushes, and slip rings. The stator cooling is achieved by the water jacket built into the stator frame and the rotor cooling is greatly simplified, merely relying on nature ventilation. The simply rotor cooling method is made possible because of much reduced losses and heat generation: no copper losses and only about half of the total iron losses. The testing of the DFBM has focused on five major aspects: i) No-load induced voltages, ii) iron losses, ii) grid connection with doubly fed control modes, iv) torque- power capabilities and v) energy efficiency over the designed speed-torque region.
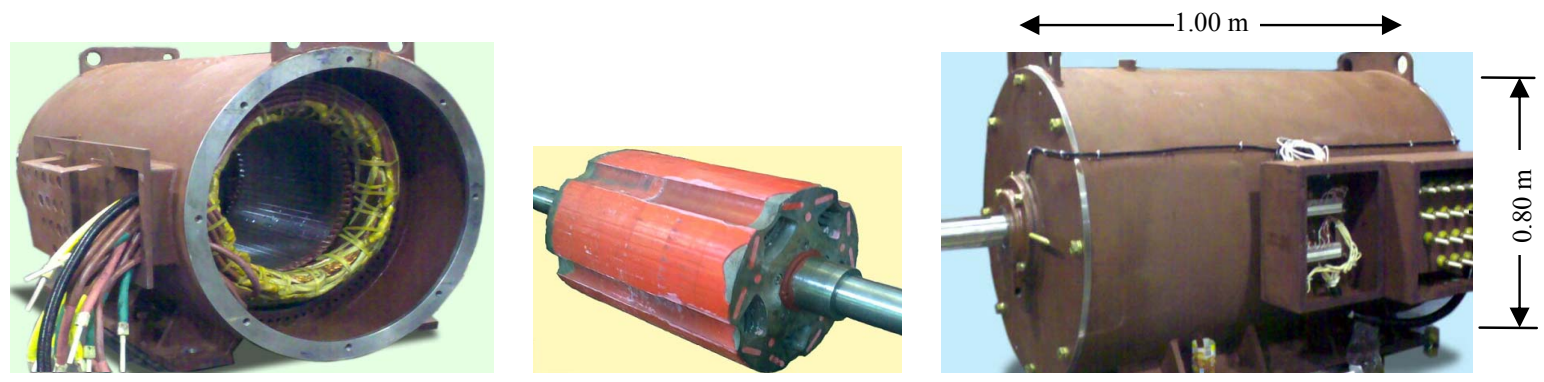

Fig. 8 Stator, rotor and total assembly of DFBM

Experimental testing concerning i) verifies the magnetizing characteristics of the designed DFBM and the results are shown in Figs. 9 (a) for the two winding voltages, and (b) excitation current in one and induced voltage in another set of stator windings

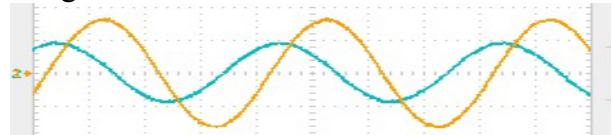

(a) blue: $\mathrm{v}_{1}=405 \mathrm{v}\left(\right.$ peak), yellow: $\mathrm{v}_{2}=228 \mathrm{v}($ peak)

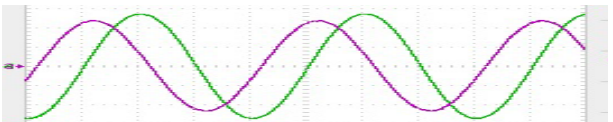

(b) purple: $\mathrm{v}_{1}=244 \mathrm{v}\left(\right.$ peak), green: $\mathrm{i}_{2}=55.2 \mathrm{~A}$

Fig. 9. v-i waveforms in no-load condition
The tested magnetizing curve as a function of excitation current is shown in Fig. 10. As shown in the figures, the induced voltages are in very good sine waveforms and in very good agreement with the theoretical results shown in Fig. 5.

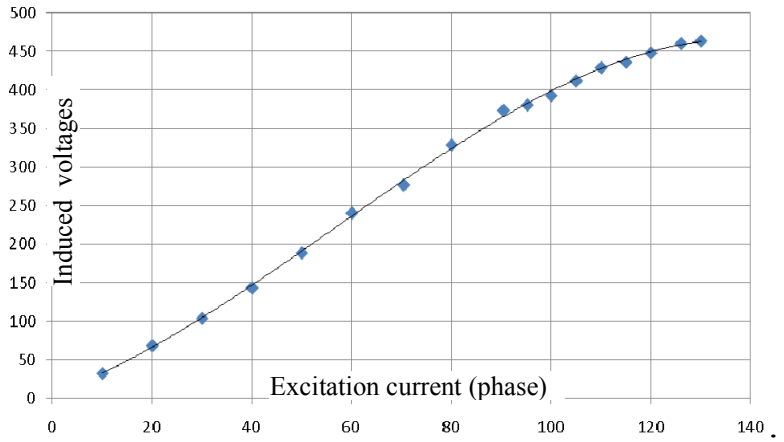

Fig. 10 Measured magnetizing curve of DFBM 
For experimental testing concerning iron losses in ii), firstly the DFBM is driven by a dynamometer to the synchronous speed of 600rpm without exciting either sets of stator windings, and input power to the shaft measured. Then, maintaining the same speed as in the first step, one set stator windings are excited with controllable current over a predetermined range, corresponding to the specified induced voltage range. For this voltage range, both input power and induced voltages are recorded. Based on the difference of input power in the first and second steps, we obtain the iron losses as a function of operation voltages. As shown in Fig. 11 , corresponding to the operation voltage range, the iron losses of the DFBM is in a range from several hundred watts to as high as $3.5 \mathrm{kw}$, depending on the operation voltages.

For experimental testing concerning iii), grid connection is made to one set stator windings.

\section{voltage - yellow traces $(100 \mathrm{v} /$ div $)$}

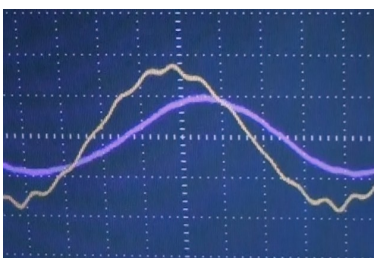

a) Lagging P. F.

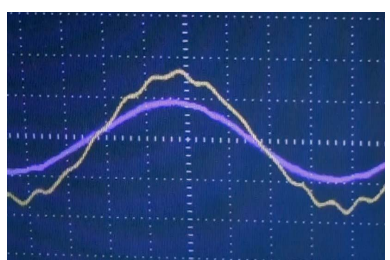

b) Unity P. F.

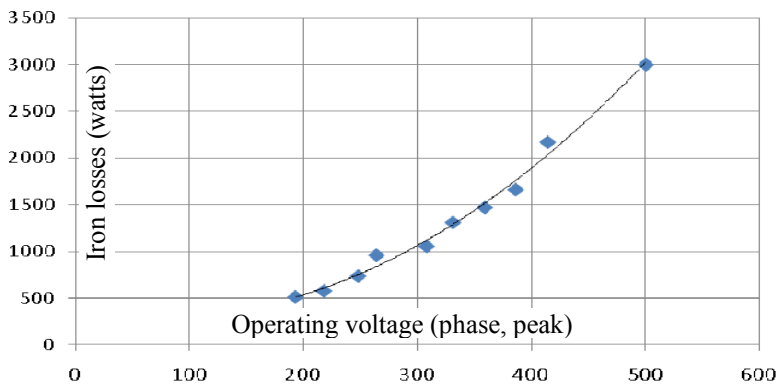

Fig. 11 Measured iron losses in no-load conditions

Control is made to the other set of stator windings through the back-to-back converter in a partial rating of the overall system and control algorithm applied. The recorded current and voltage waveforms are shown in Figs. 12 (a) through (d).

Figs. 12 Voltage and current waveforms in loaded Conditions

As demonstrated by the waveforms, the DFBM can continuously achieve decoupled reactive and active power control through the vector control of current components, $\mathrm{i}_{\mathrm{d} 2}$ and $\mathrm{i}_{\mathrm{q} 2}$, for lagging, leading, and unity power factor operations. The controlled active power through the back-to-back inverter is only a fraction of the total rating of the system, resulting in a low-cost DFBM system.

For experimental testing concerning iv) and v), the power capabilities and energy efficiency, the DFBM is loaded with active power on the two sets of stator windings, and input and output power recorded. The power capability testing is conducted in 5 levels of torque $(400,800,1200,1600$, and $2000 \mathrm{Nm}$ ), combined with 4 levels of speeds (400, 600, 900, and $1200 \mathrm{rpm}$. At each operating point, the energy efficiency is examined. As indicated by the results the designed DFBM machine is fully capable of rated power $(200 \mathrm{kw})$ and can also be $25 \%$ over the rated power $(250 \mathrm{kw})$.

The energy efficiency of the DFBM is plotted in Fig. 13 by the equal-efficiency contours. Clearly shown is that $90 \%$ operating points in the regime has efficiency higher than $92 \%$, $70 \%$ operating points higher than $94 \%$, and $35 \%$ operating points higher than $96 \%$ including the rated and over-rated power operating points. The torque and power capability of the designed DFBM is very satisfactory and energy efficiency is record-breaking high in the family of similar machines.

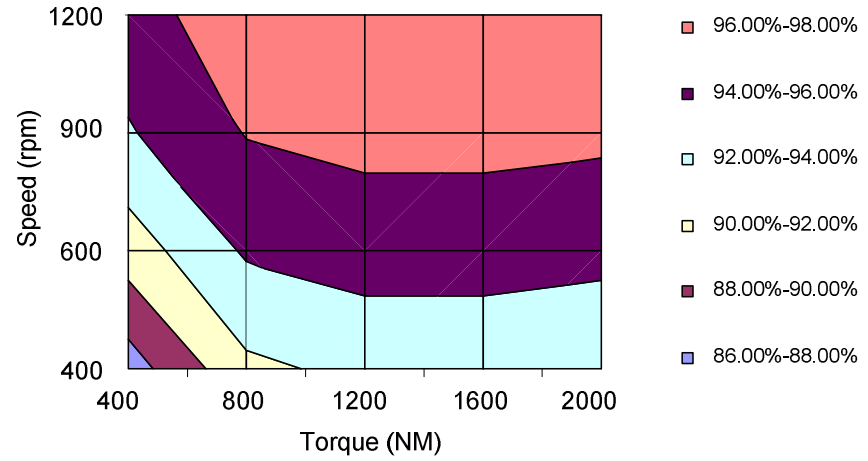

Fig. 13 Measured efficiency contour map in loaded conditions

Accoustic noise and vibration has been observed for the DFBM built. At a fixed rotor speed, the noise and vibration intensities are proportional to the voltage levels, instead of load levels. Therefore, the noise and vibration can be attributed to the magnetic flux levels in the DFBM field, a direct hint that the unsymmetic magnetic field distribution might be responsible. It can be derived that if the magnetic 
flux distribution of the field is improved to symmetric, the noise and vibration problems may be elleviated.

\section{CONCLUSIONS}

Starting with a brief review of the 100-year-old history of DFBM evolution, this paper presents our latest investigation of design and advanced control of DFBM. The challenges of designing a high-efficiency DFBM and system are discussed and the effective solutions to the optimal design and control a high efficiency DFBM identified. Using first principles assisted by finite element analysis, an original design of DFBM and system is achieved, capable of $2000 \mathrm{Nm}$ in the speed range of 400-1200rpm with a frame size comparable to that of a brush type doubly fed induction machine. The designed machine is built and tested, and the experimental results are in very good agreement with the design objectives. The most successful results include the DFBM power capability more than $25 \%$ over the desined value and energy efficiency higher than $94 \%$ covering $75 \%$ designed torquespeed regime. These theoretical and experimental results represent game-changing achievement of doubly fed brushless technology.

The following can be concluded from our original design of the DFBM: a) the rotor design and the selection of pole number combination are keys to the power density and efficiency, competing with it brush type counterpart; b) in addition to the features of robustness and maintenance-free, the DFBM is a controlled machine and can achieve decoupled control of active and reactive power by vector control; and c) research attentions are needed to improve magnetic field distribution to reduce noise and vibrations in DFBM machines. Based on the achieved results, we strongly contend that DFBM technology, with its intrinsic advantages of brushless and doubly fed operation modes, is highly potential in today's green energy economy, especially for wind turbine generators. Also needed is to establish systematic design procedures for DFBM design so that optimal design in various sizes, power ratings, and for different applications can be accomplished effectively to meet the market demand. As a final note, the major losses of DFBM are on the stator side, more aggressive cool methods and new conducting materials such as super conducting coils can be applied to DFBM in an easier manner. The DFBM technology seems very potential to pursue much further.

\section{References:}

1. L.J. Hunt, "The Cascade Induction Motor," J. IEE Vol. 52, pp. 406-426, 1914

2. F. Creedy, "Some Developments in Multi-Speed Cascade Induction Motors", J. IEEE, vol59, pp. 551-552, 1921.
3. Boardman, G.; Zhu, J.G.; Ha, Q. "Dynamic and steady state modeling of brushless doubly fed induction machines", Electrical Machines and Systems, 2001. ICEMS 2001. Proceedings of the Fifth International Conference on, Volume 1, 18-20 Aug. 2001 Page(s):412 - 416

4. A.R.W. Broadway, "Cageless Induction Motor", Proc. IEE, V O 1 18, pp. 1593-1600, 1971.

5. A.R.W. Broadway and G. Thomas, "Brushless Cascade Alternator", Proc. IEE, vol 121, pp. 1529-1535, 1974.

6. A. Kusko and C.B. Somuah, "Speed Control of a SingleFrame Cascade Induction Motor with Slip-Power Pump Back", IEEE Trans. on Industry Applications, vol. IA-14, April 1978, pp. 97-105.

7. C.J. Heyne and A.M. El-Antably, "Reluctance and DoublyExcited Reluctance Motors", Final Report, Oak Ridge National Laboratories, Report ORNUSUB/81-95013, 1981

8. L. Xu, F. Liang and T.A. Lipo "Transient Model of a Doubly Excited Reluctance Motor", IEEE, Transactions on Energy Conversion. Vol. 6, No. 1, March 1991, pp. 126-133

9. Ruqi Li, Alan Wallace, Rene Spee, Yixin Wang "Two-Axis Model Development of cage-rotor Brushless of Doubly Fed Machine", IEEE Transactions on Energy Conversion, Vol. 6, No. 3, September $1991453-460$

10. Betz, R.E. Jovanovic, M.G.The "brushless doubly fed reluctance machine and the synchronous reluctance machinea comparison," IEEE Transactions on Industry Applications, Jul/Aug 2000, Volume: 36, Issue: 4, pp 1103-1110

11. Fengxiang Wang and Fengge Zhang Longya Xu "Parameter and Performance Comparison of Doubly-Fed Brushless Machine with Cage and Reluctance Rotors", IEEE Transactions on Industry Applications, Sep/Oct 2002, Volume: 38, Issue: 5, pp: 1237- 1243

12. L. $\mathrm{Xu}$ and F. Wang, "Comparative study of magnetic coupling for a doubly fed brushless machine with reluctance and cage rotors", Thirty-Second IAS Annual Meeting, IAS '97., Conference Record of the 1997 IEEE Vol.1, Oct. 1997 pp.326-332

13.L. Xu, "Analysis of a Doubly-Excited Brushless Reluctance Machine by Finite Element Method", IEEE Industry Application Society Annual Meeting, 1992, Houston, vol.1, pp.171-177.

14. Longya $\mathrm{Xu}$ and Huijuan Liu, "Rotor Pole Number Studies for Doubly Excited Brushless Machine", IEEE Energy Conversion Congress and Exposition (ECCE2009), San Jose, California, USA, September 20-24, 2009, Proceedings, pp. 208-213 